\title{
Angewandte
}

Supporting Information

(c) Wiley-VCH 2009

69451 Weinheim, Germany 


\section{Multicompartmental Microcylinders}

Srijanani Bhaskar, Jonathon Hitt, Sei-Won Laura Chang and Joerg Lahann*

(a)

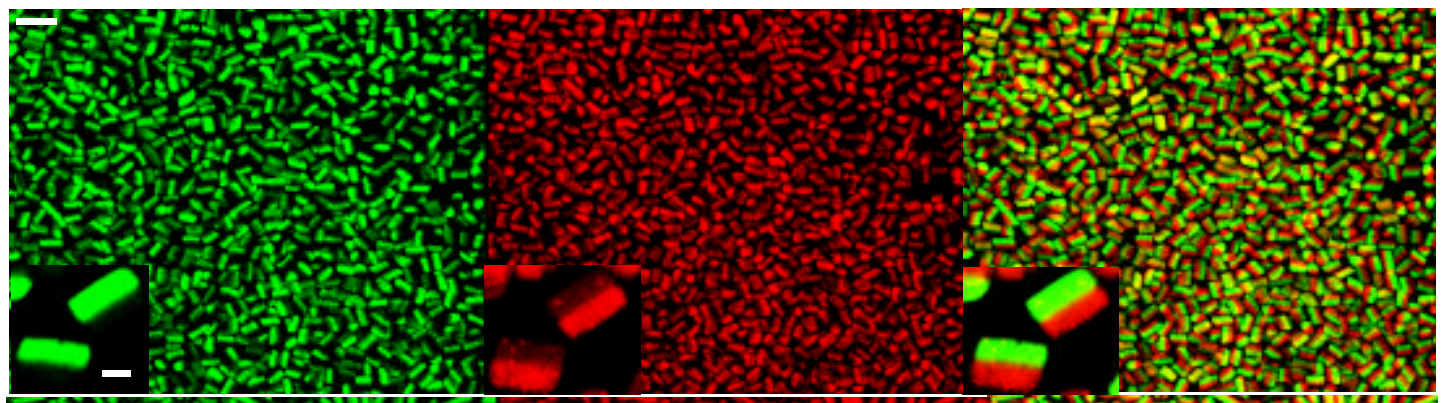

(b)

(c)

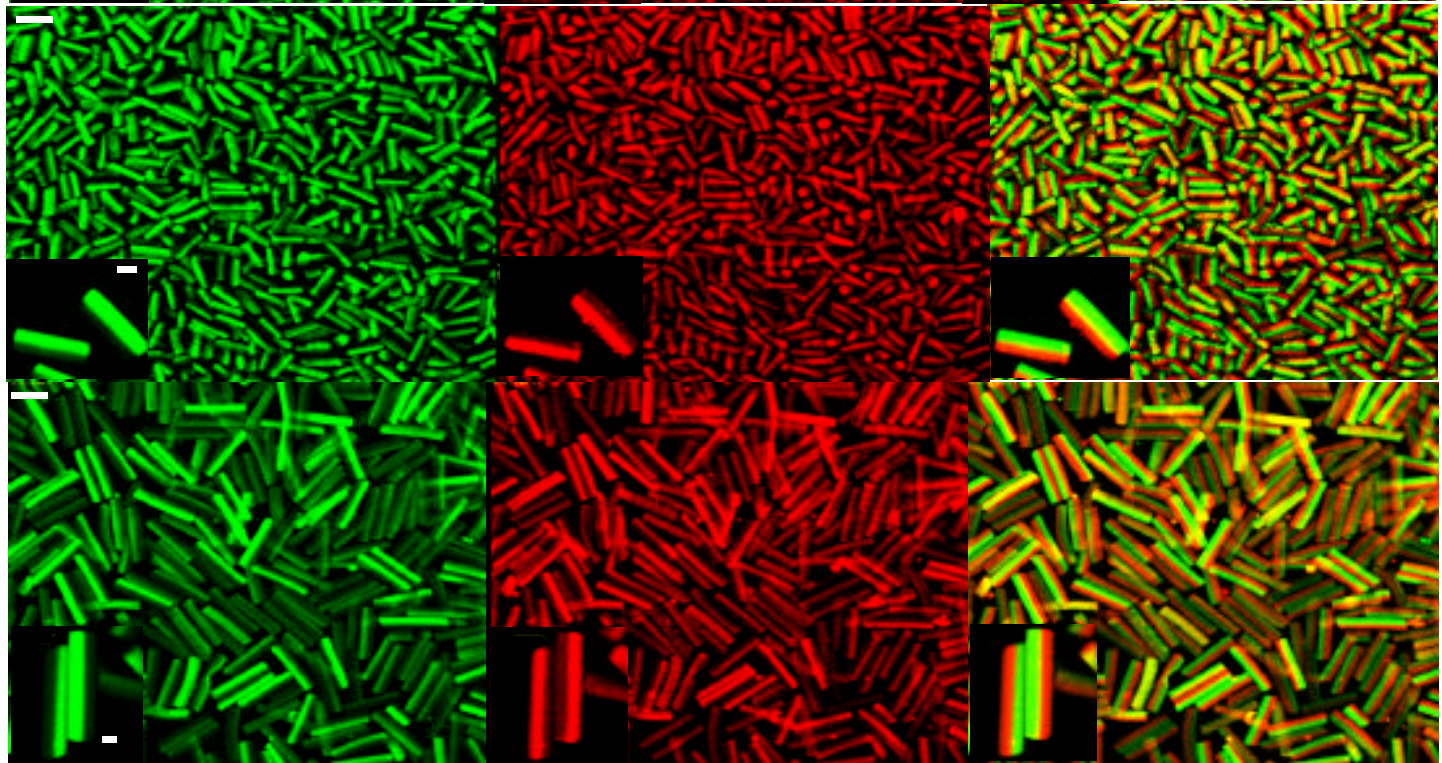

Figure S1. Individual compartment visualization of bicompartmental microcylinders with varying aspect ratios (A. R). The difference in $A$. $R$ arises from sectioning at different lengths. Green and red fluorescence images (representing PTDPV and ADS306PT) depicting individual compartments are shown, followed by their overlay which shows the entire microcylinder. a) length $20 \mu \mathrm{m}, \mathrm{A}$. $\mathrm{R}$ $=1.3$. b) length $39 \mu \mathrm{m}, \mathrm{A} . \mathrm{R}=2.6, \mathrm{c}$ ) length $80 \mu \mathrm{m}, \mathrm{A} . \mathrm{R}=4.6$. Inlays show high magnification images. Scale bars represent $50 \mu \mathrm{m}$ for outer images and 10 $\mu \mathrm{m}$ for inlays. 
Figure S2. (a) Size distribution analysis for cylinder diameter. Diameters were determined from Figure 1b using 'Image J' software.

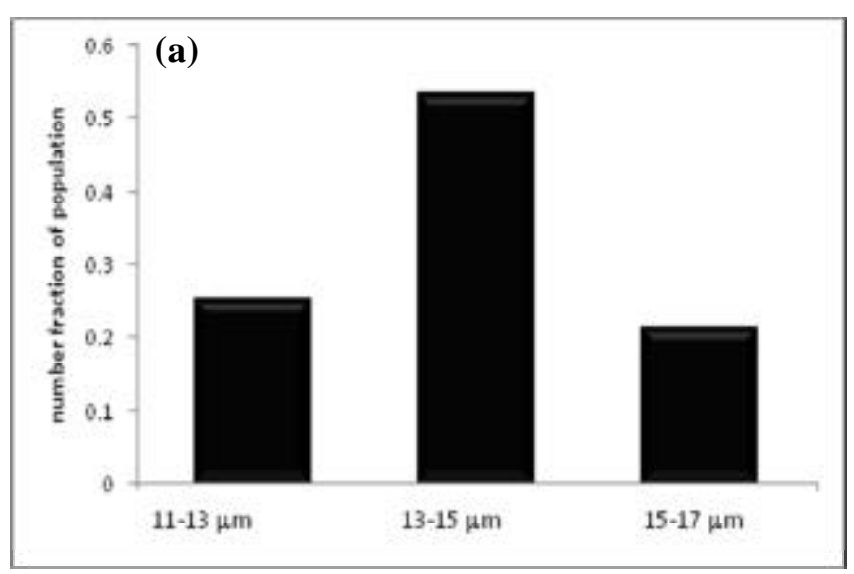

(b)

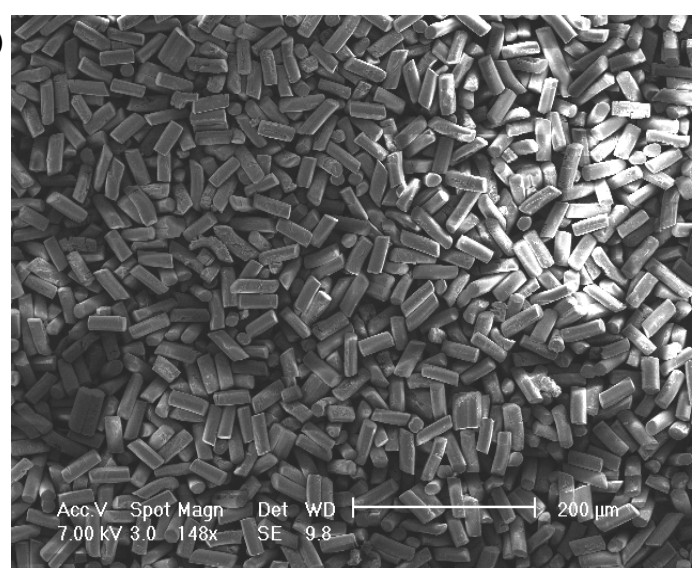




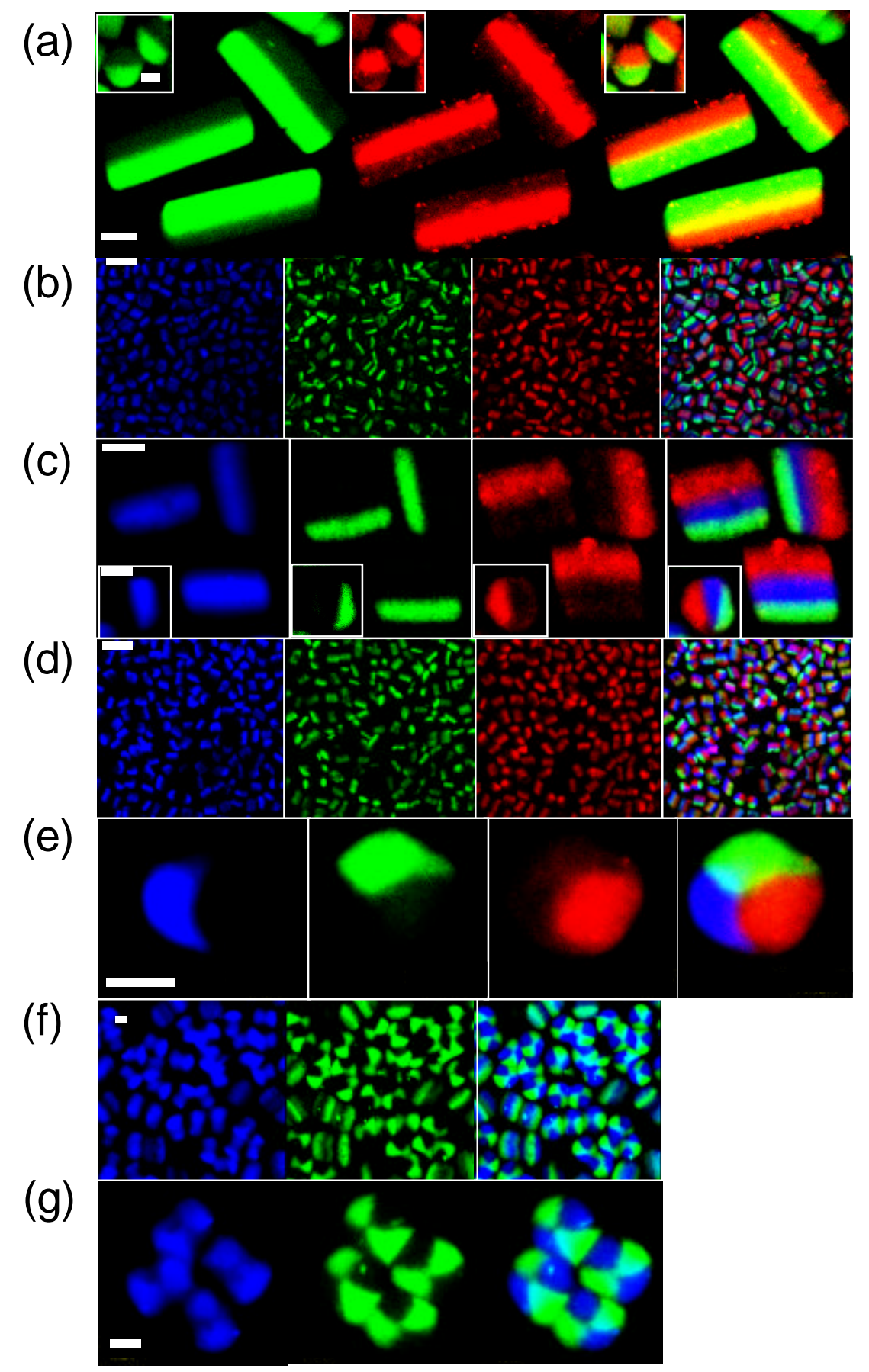

Figure S3. Visualization of distinct compartments of microcylinders via CLSM. Individual blue, green and red fluorescence images (representing MEHPPV, PTDPV and ADS306PT) are shown, followed by their overlay. a) Bicompartmental microcylinders. Inset shows cross sectional view for clear visualization of compartments. b), c) low and high magnification images of tricompartmental microcylinders formed from fibers which were fabricated by co-spinning from a sequential arrangement of three outlet streams. Inlay of (c) shows a cross sectional view. d), e) low and high magnification micrographs of 'pie' shaped microcylinders resulting from fibers fabricated via a trigonal nozzle configuration. f), g) four compartmental microcylinders exhibiting a 'bow-tie' type compartmentalization of blue and green dyes, which upon overlaying yield rosette shaped microcylinders. These were made from a square shaped macroscopic nozzle positioning with opposite quadrants loaded with the same dye (blue and green). Scale bars denote $50 \mu \mathrm{m}$ for (b) and (d), and $10 \mu \mathrm{m}$ for all others. 


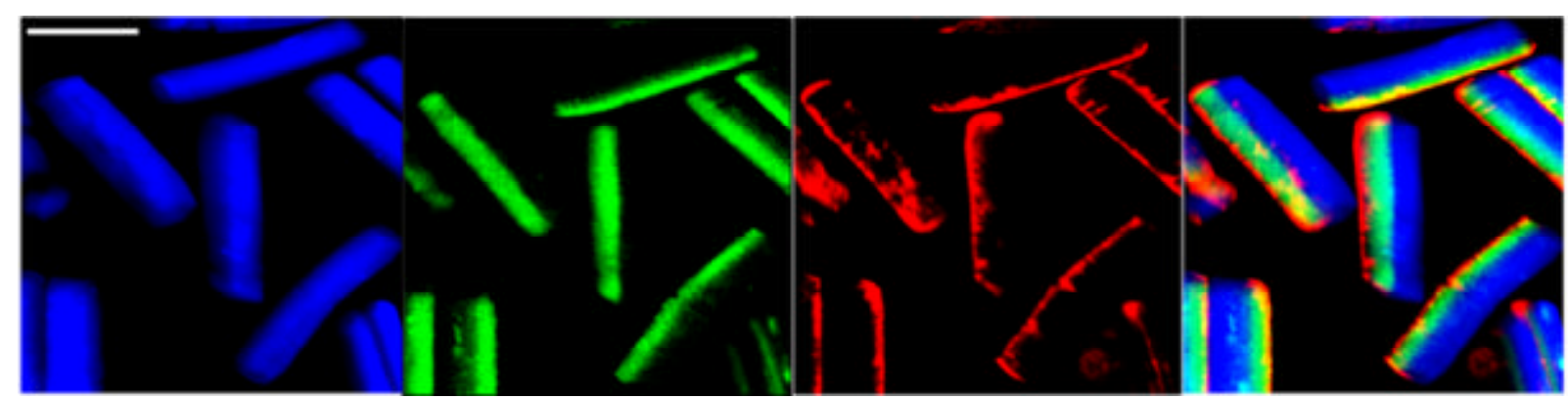

Figure S4. Imaging of individual compartments of selectively phase modified microfibers. Four-compartmental microfibers with one compartment three folds smaller than the other were prepared via sectioning of tetracompartmental fibers from a square shaped macroscopic nozzle configuration where three solutions were loaded with MEHPPV (blue fluorescence) and the fourth with PTDPV (green fluorescence). Acetylene functionalized PLGA, poly[lactide-co-(propargyl glycolide)], was also incorporated into to the fourth solution. The resulting microcylinders were then reacted with azide-biotin via copper catalyzed Huisgen cycloaddition. The resulting surface biotin groups were then reacted with Alexa Fluor-633 labeled streptavidin. The red fluorescence of Alexa Fluor 633 was observed only along the periphery of the smaller compartment, indicating successful selective surface modification with streptavidin, as seen in the overlay of individual fluorescence images. Scale bar represents $50 \mu \mathrm{m}$.

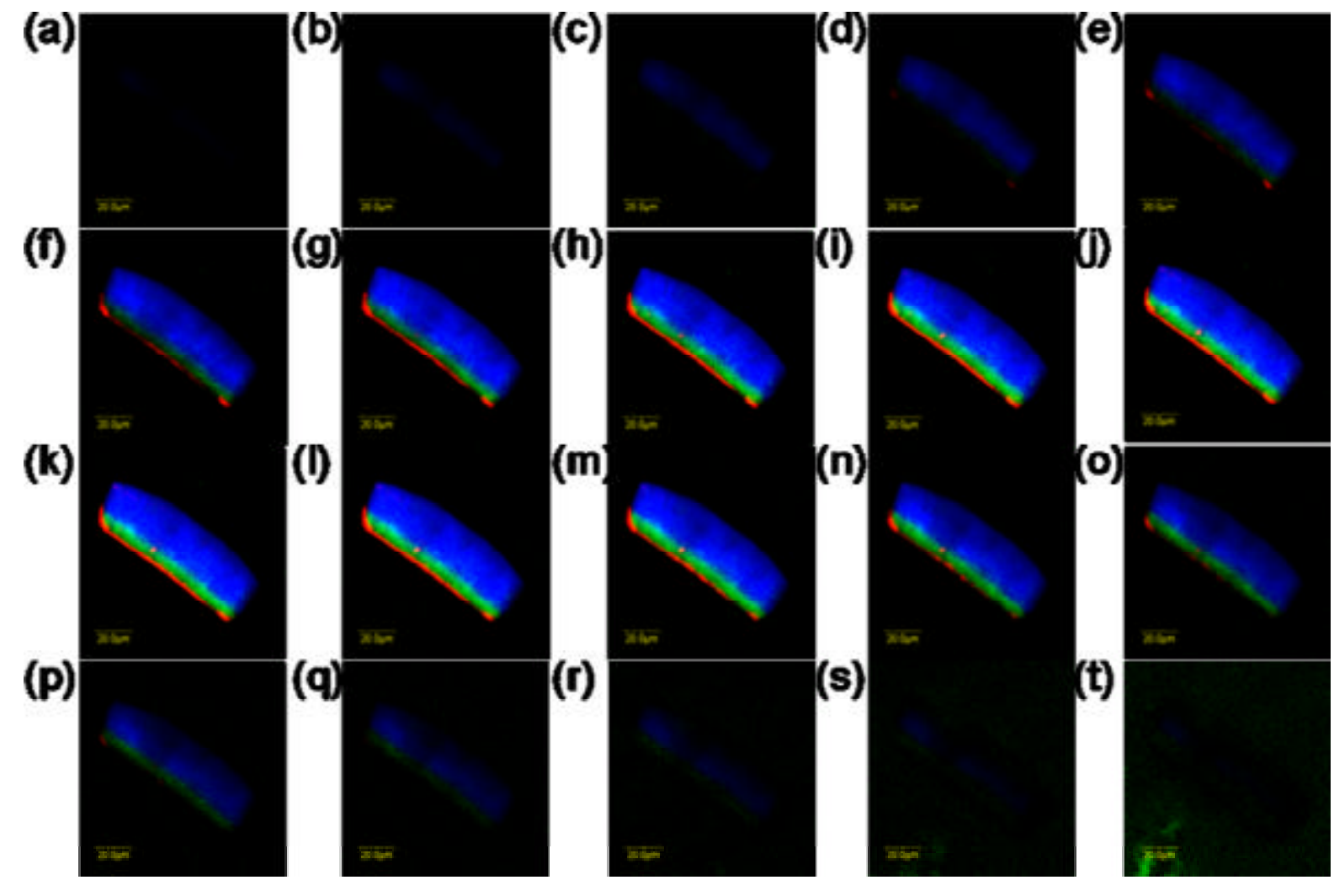

Figure S5. Z stack-CLSM micrographs of a representative tetracompartmental microcylinder, modified with Alexa-633 labeled streptavidin over one compartment, i.e. one-fourth surface only. These images were utilized for a three dimensional reconstruction of the cylinder to visualize the localization of strepatvidin in a perspective view, included as a movie. Blue, green and red fluorescence represent MEHPPV, PTDPV and Alexa-Fluor 633 respectively. The depth between successive images is $1.5 \mu \mathrm{m}$. As seen from individual sections, red fluorescence from Alexa-Fluor 633 is confined to the smaller green compartment only, at all depths. 


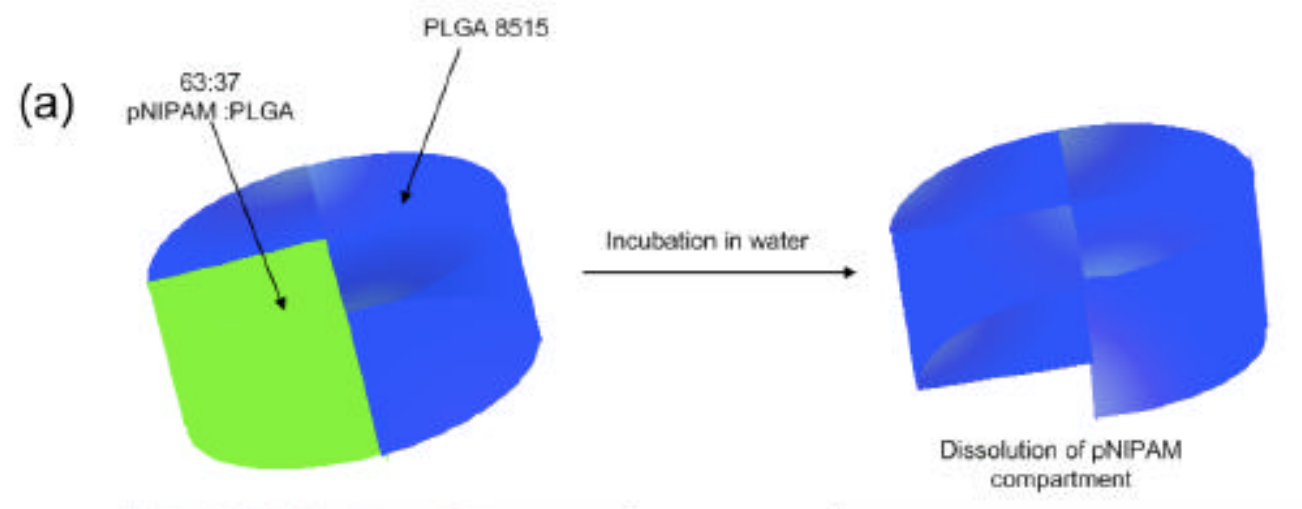

(b)
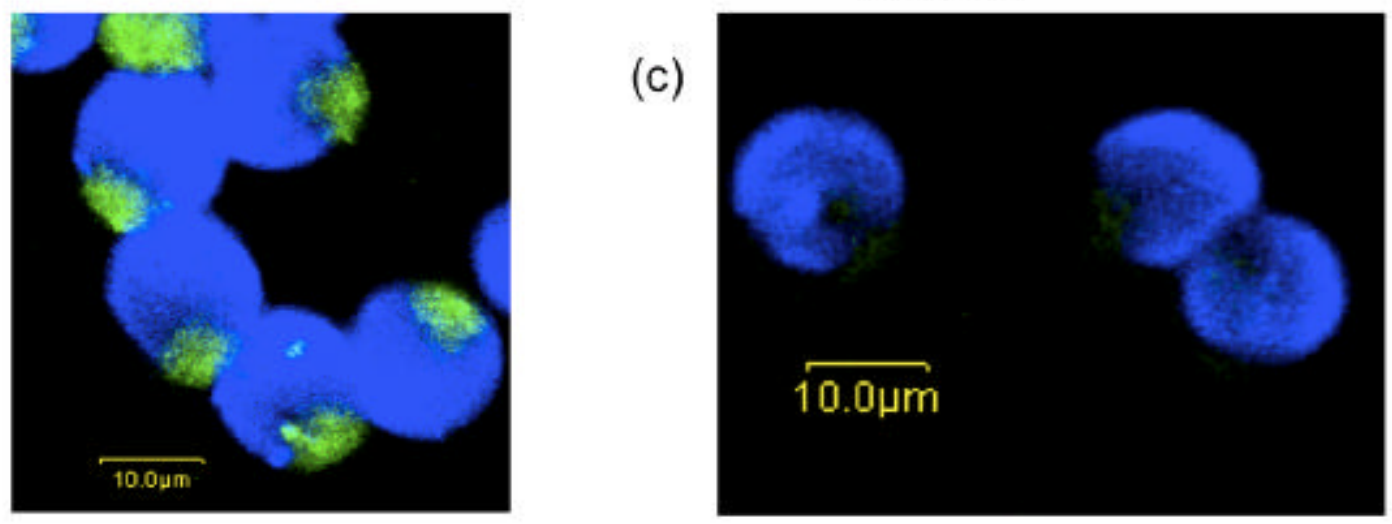

(d)

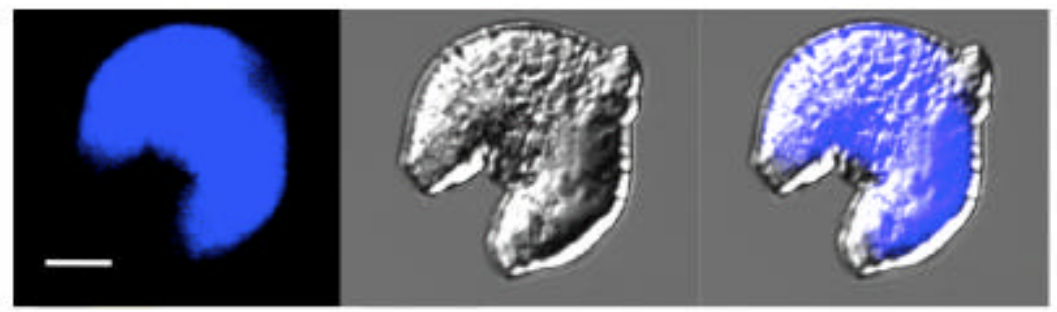

Figure S6. (a) Schematic showing fabrication of 'packman' shaped microdisks. Tetracompartmental microdisks containing PLGA85:15 in three compartments and a 63:37 w/w ratio of pNIPAM:PLGA in the fourth compartment were fabricated via electrohydrodynamic co-spinning from a square shaped needle configuration followed by microcutting. The three compartments comprising of PLGA were labeled with MEH-PPV (blue fluorescence), whereas the pNIPAM/PLGA compartment was labeled with PTDPV (green fluorescence). The microdisks had an aspect ratio of 0.25 . Incubation of the microdisks in water for 10 days led to dissolution of the pNIPAM from the quarter compartment, yielding microdisks with a 'packman' like cross-section. (b) CLSM micrographs of fiber cross section directly after co-spinning showing distinctive green fluorescence from the pNIPAM/PLGA compartment (c) CLSM micrographs of microdisks after incubation in water for 10 days, showing negligible green fluorescence, indicative of dissolution of the quarter compartment. (d) Isolated 'packman' particle visualized by CLSM and DIC microscopy shown along with the overlay of both images (from left to right). Scale bars represent $10 \mu \mathrm{m}$. 
Supporting Movie: Three dimensional visualization of a tetracompartmental micro cylinder modified with streptavidin on one-fourth of its surface area only. A Z stack analysis was performed on a representative cylinder along its longitudinal axis at a section depth of $1.5 \mu \mathrm{m}$. The Z-series was reconstructed (using Imaris ${ }^{\mathrm{TM}}$ ) to afford a perspective view of the cylinder. The cylinder was rotated in 3D space and snapshots at different rotation angles were compiled into a movie. Blue, green and red fluorescence indicate MEH-PPV, PTDPV and Alexa Fluor 633 streptavidin respectively. As seen in the longitudinal view in major portions of the movie, red fluorescence is present throughout the surface of the smaller compartment. The cross sectional view from 0:12 to $0: 15$, followed by high magnification examination in $0: 18$ to $0: 27$ clearly indicates that the biotin binding to the fiber surface is well confined and hence highly specific. The side of each square in the grid represents $10 \mu \mathrm{m}$. 